The AstrophysiCal Journal, 341 : L71-L74, 1989 June 15

(C) 1989. The American Astronomical Society. All rights reserved. Printed in U.S.A.

\title{
BIASED COLD DARK MATTER THEORY: TROUBLE FROM RICH CLUSTERS?
}

\author{
AUgust E. EVRaRD \\ Department of Astronomy, University of California, Berkeley \\ Received 1989 February 21 ; accepted 1989 April 3
}

\begin{abstract}
The expected population of rich clusters in a cold dark matter (CDM)-dominated universe is extremely sensitive to the spectrum normalization, the inverse of which defines the bias parameter $b$. Recent observations of three high-velocity dispersion clusters at redshifts $z \approx 0.5$ indicate an alarmingly low value of $b$ for the theory. If taken at face value, the observations imply an unbiased model, $b \simeq 1$. However, this value of $b$ predicts roughly 10 times as many low-redshift clusters $(z<0.1)$ as are observed. The low-redshift data favor values in the range $b \simeq 1.5-1.9$. It is argued that poor statistics and the uncertainty in recovering intrinsic velocity dispersions from redshift measurements preclude a reliable determination of $b$ at present. A survey of cluster X-ray temperatures complete to moderate redshifts would prove invaluable in deciding this issue.
\end{abstract}

Subject headings: clusters: open - cosmology

\section{INTRODUCTION}

One of the most appealing aspects of the cold dark matter (CDM) model is the small number of free parameters in the theory. If one assumes that physics of the early universe produces a constant curvature spectrum with random phases, there remain only three parameters to determine the theorythe density parameter $\Omega$, the expansion rate $h=H_{0} / 100 \mathrm{~km}$ $\mathrm{s}^{-1} \mathrm{Mpc}^{-1}$, and the present linearly extrapolated amplitude $\sigma_{\rho}(M)$ for the rms fluctuations in mass within a sphere which, on average, contains mass $M$. Theoretical prejudice argues for $\Omega=1$, leaving only $h$ and $\sigma_{\rho}$ to be quantified.

The first determination of these parameters by Davis et al. (1985) required a biased model of galaxy formation to reproduce the observed slope of the galaxy correlation function and obtain acceptable values $h \gtrsim 0.5$ for the Hubble constant. In the biased model, the fluctuations in galaxies will in general be different from those of the underlying mass (Bardeen et al. 1986). This difference is conventionally expressed by the bias parameter

$$
b \equiv \frac{\sigma_{g}\left(8 h^{-1} \mathrm{Mpc}\right)}{\sigma_{\rho}\left(8 h^{-1} \mathrm{Mpc}\right)}=\frac{1}{\sigma_{\rho}\left(8 h^{-1} \mathrm{Mpc}\right)},
$$

which is the ratio of galaxy to mass fluctuations in a sphere of radius $8 h^{-1} \mathrm{Mpc}$, corresponding to a mass scale $M=6 \times 10^{14} h^{-1} M_{\odot}$, roughly the mass scale of rich clusters. Since the level of fluctuations in galaxy counts on this scale is observed to be unity, $\sigma_{g}\left(8 h^{-1} \mathrm{Mpc}\right)=1$ (Davis and Peebles 1983), $b$ can be interpreted simply as a measure of the inverse of the spectrum normalization on the mass scale of rich clusters.

The value of $b$ is of central importance to the CDM model. It normalizes the level of fluctuations in the microwave background at all scales. On small scales, it controls such observables as pairwise galactic velocities, mass-to-light ratios of groups and clusters, and the abundance of halos with given circular velocities. Many of the successes of the model on galaxy group and smaller scales (Frenk et al. 1988) will evaporate if $b \simeq 1$ rather than $b \simeq 2$. The redshift of the "epoch of galaxy formation" scales inversely with b (Evrard 1989b), affecting interpretation of galaxies observed at high redshift. On large scales, the amplitude and statistics of linear flow velocities are sensitive to $b$ (Kaiser and Lahav 1989; Bertschinger and Juszkiewicz 1988; Gorski et al. 1989).

The abundance and properties of rich clusters should provide a sensitive measure of the spectrum normalization, since in the model they correspond to objects lying in the tail of an initially Gaussian distribution with variance $\sigma_{\rho}(M) \propto b^{-1}$. Recently, Gunn (1989), in collaboration with Dressler, has released velocity dispersions for several high-redshift clusters. Among them are some of the highest velocity dispersion systems ever measured. The purpose of this Letter is to investigate what these observations tell us about the CDM normalization or, equivalently, the "level of biasing" $b$.

\section{THE MODEL}

To test CDM model predictions against observations, we need to calculate $n(v, z) d \ln v d z$, the abundance per unit redshift of clusters with one-dimensional velocity dispersion $v$. The approach taken here is to start with the Press-Schechter (1974) formula which predicts number density as a function of mass at a given redshift

$$
\begin{aligned}
n(M, z) d \ln M=-\sqrt{\frac{2}{\pi}} \frac{\rho_{0}}{M} \frac{d \log \sigma_{\rho}}{d \log M} v_{z}(M) \\
\times \exp \left[-\frac{v_{z}^{2}(M)}{2}\right] d \ln M,
\end{aligned}
$$

where $v_{z}(M)=1.68(1+z) / \sigma_{\rho}(M)$. The predictions of equation (3) agree well with the multiplicity functions determined by $N$-body experiments over a wide range of initial conditions (Efstathiou et al. 1988). For simplicity, we use a power-law approximation to the CDM spectrum $\sigma_{\rho}(M)=1.05 b^{-1}$ $M_{15}{ }^{-1 / 3}$, where $M_{15}=M / 10^{15} M_{\odot}$ which is accurate to better than $5 \%$ for $0.5<\log _{10} M_{15}<1$, the mass range of the richest clusters. The number of clusters per comoving cubic megaparsec is then ( $h=0.5$ is assumed hereafter)

$$
\begin{aligned}
n(M, z) d \ln M= & 2.95 \times 10^{-5} b(1+z) M_{15}{ }^{-2 / 3} \\
& \times \exp \left\{-0.5\left[1.60 b(1+z) M_{15}{ }^{1 / 3}\right]\right\} d \ln M .
\end{aligned}
$$

We now use a relation between velocity dispersion and mass to predict abundance as a function of velocity dispersion. 


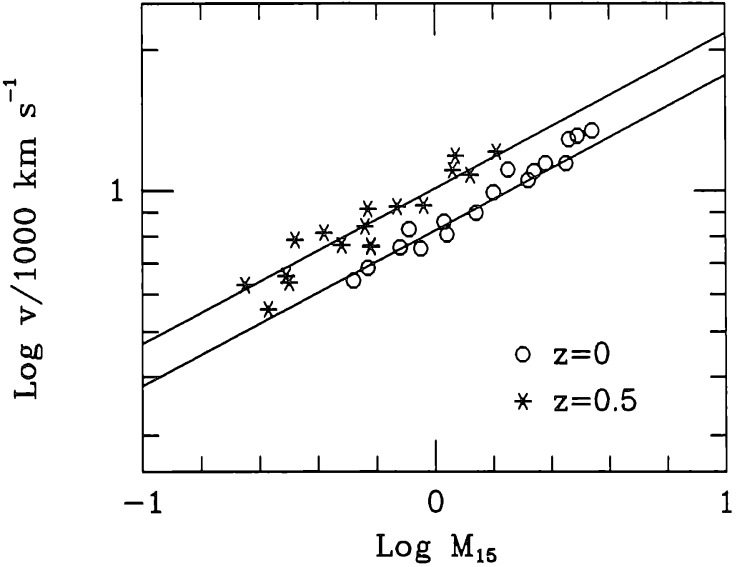

FIG. 1.-The velocity-mass relation determined by $N$-body simulations is compared with the theoretical expectation, eq. (4), using $f=0.9$ at redshifts $z=0.5$ (asterisks) and $z=0$ (circles). The mass scale $M$ in the experiments is defined as the mass enclosed within a region of overdensity $\delta \rho / \rho=170$ from the cluster center. This corresponds to the overdensity of the mass scale just collapsed in the analytic model. Note the $\sim 10 \%$ residual scatter in the numerical results about the analytic estimate.

Using a spherical shell approximation (Gunn and Gott 1972) in which mass shells contract by a factor of 2 from their maximum expansion values and "virialize," we obtain

$$
v=f\left(727 \mathrm{~km} \mathrm{~s}^{-1}\right)(1+z)^{1 / 2} M_{15}{ }^{1 / 3},
$$

where $v$ is the one-dimensional velocity dispersion and $f$ is a "fudge factor" which accounts for deviations of real clusters from the simple model. Comparison of this expression with results of $N$-body experiments is shown in Figure 1. The simulations are $\mathrm{CDM}$ realizations designed explicitly for the purpose of studying the internal properties of rich clusters (Evrard 1989c; see also Evrard 1989a). The numerical results are in good agreement with the analytic model using $f=0.9$. More importantly, the simulations show a $\sim 10 \%$ scatter about the analytic line.

The $v-M$ relation is used with equation (2) to produce an abundance expression $n(v, z) d \ln v$ which, when integrated, gives $\mathcal{N}(v, z)$, the expected number density of clusters with velocity dispersion greater than $v$

$$
\mathscr{N}(v, z)=5.28 \times 10^{-5} b^{3}(1+z)^{3} \Gamma[-1, t(v, z)] \mathrm{Mpc}^{-3},
$$

where $t(v, z)=1.28 b^{2}(1+z)\left(v / 650 \mathrm{~km} \mathrm{~s}^{-1}\right)^{2}$ and $\Gamma[-1, t(v, z)]$ is an incomplete gamma function.

This equation assumes a one-to-one correspondence between velocity $v$ and mass $M$. In reality, there is bound to be some dispersion about this mean relation. The scatter may be partly intrinsic, arising from different dynamical histories of clusters of a given mass (Fig. 1). Further scatter will arise from observational error. An estimate of the cluster velocity dispersion based on $N_{z}$ redshifts will differ from the true value by some amount $\delta v$. In the ideal case of sampling an isotropic Gaussian velocity distribution with true dispersion $v$, the expected error $\delta v / v$ without contamination will be Gaussian distributed with variance $1 /\left(2 N_{z}\right)^{1 / 2}$ for $N_{z} \gtrsim 30$. This ideal case represents a lower bound on the actual error, since it ignores contamination by projected galaxies not physically associated with the virialized portion of the cluster.

In the model, these uncertainties can be incorporated by convolving with a probability distribution $P(\delta v)$ for deviations of the measured dispersion from the mean relation. The number density of clusters expected with observed velocity dispersion greater than $v$ can be expressed as

$$
\mathscr{N}_{\text {obs }}(v, z)=\int d \delta v P(\delta v) \mathcal{N}(v+\delta v, z)
$$

Finally, we can use this expression to estimate the expected number of objects on the sky with velocity dispersions greater than $v$ lying within a redshift $z$

$$
\begin{aligned}
N(v, z)=5.73 \times & 10^{8} b^{3} \int_{1}^{1+z} d y y^{-3 / 2}\left(1-y^{-1 / 2}\right)^{2} \\
& \times \int d \delta v P(\delta v) \Gamma[-1, t(v+\delta v, y-1)] .
\end{aligned}
$$

\section{RESULTS}

The sensitivity of the predicted abundances $N(v, z)$ to velocity cutoff and assumed scatter in the $v-M$ relation is shown in Figure 2. The solid line shows the results of using the mean relation, equation (4), with no dispersion. Note the sensitivity of the abundance to the limiting velocity $v$ : the density drops four orders of magnitude as $v$ changes by only a factor of 2 . The dot-dashed and dashed lines assume a Gaussian distribution of uncertainties $P(\delta v)$ with variance $0.125 v$ and $0.20 v$, respectively. The effect of including dispersion with $10 \%-20 \%$ amplitude can be dramatic at large velocities-a direct consequence of working on the tail of an exponentially sensitive distribution.

\section{a) High-Redshift Analysis}

Gunn (1989) has listed three clusters with velocity dispersions $v>1350 \mathrm{~km} \mathrm{~s}^{-1}$ (based on $\sim 30$ redshifts per cluster) lying within a redshift $z \simeq 0.5$. They are 9HF $\alpha \beta(z=0.391$, $\left.v=1600 \mathrm{~km} \mathrm{~s}^{-1}\right), \mathrm{Cl} 0024+16\left(z=0.407, v=1350 \mathrm{~km} \mathrm{~s}^{-1}\right)$, and $\mathrm{Cl} 0016+16\left(z=0.546, v=1600 \mathrm{~km} \mathrm{~s}^{-1}\right)$. The cluster 3C $295\left(z=0.467, v=3000 \mathrm{~km} \mathrm{~s}^{-1}\right)$ is labeled by Gunn as "dynamically peculiar"-probably a collection smaller clusters seen in projection - and so is not included here.

In matching to model predictions, it is necessary to know what fraction of the sky was surveyed to this depth in order to

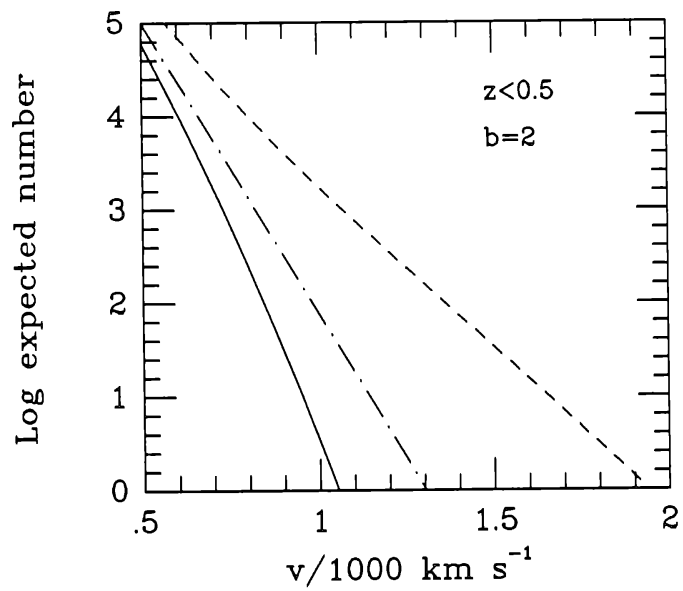

FIg. 2.-The number of clusters expected within a redshift $z=0.5$ having observed velocity dispersions greater than $v$ is shown for the case $b=2$. The solid line assumes that observed velocity dispersions follow the mean $v-M$ relation with no scatter. The dot-dashed and dashed lines assume Gaussian scatter with variance $0.125 v$ and $0.20 v$, respectively. The expected number of clusters is clearly very sensitive to both the velocity cutoff $v$ and the assumed level of scatter. 
uncover the underlying abundance on the whole sky. One of the clusters $(9 \mathrm{HF} \alpha \beta)$ is from the deep survey of Gunn, Hoessel and Oke (1986) which covered about $40 \mathrm{deg}^{2}$ and is complete to $z \simeq 0.5$. The other two were serendipitous findings, one $(0016+16)$ arose in the deep pencil beam surveys conducted by Koo and Kron (1987) which covered only a few square degrees of sky. Although it is difficult to estimate precisely the sky coverage for the accidental clusters, it is probably $\lesssim 10 \mathrm{deg}^{2}$ (H. Spinrad, private communication). This tells us that the three clusters were discovered over a total area of $\sim 50 \mathrm{deg}^{2}$. Since there are $\sim 40,000 \mathrm{deg}^{2}$ on the sky, the observations imply that there are $N \simeq 2500$ clusters with velocity dispersions $v>1350 \mathrm{~km} \mathrm{~s}^{-1}$ and redshifts $z<0.5$ over the entire sky. The statistical uncertainty in this number is impossible to quantify, but only the most optimistic would believe it to better than a factor of 2 .

Figure $3 a$ shows the model estimates derived from equation (7) as a function of the bias parameter $b$. Different line types indicate results for different assumed amounts of scatter in the $v-M$ relation-0, $12.5 \%$, and 20\%-as in Figure 2. The two long dashed lines delimit a range a factor of 2 on either side of the number determined above from the observations, $N \sim 2500$.

The expected number predicted by the model is clearly very sensitive to the value of $b$. However, it is also sensitive to the amount of assumed $v-M$ scatter. It is unlikely that the combined effects of intrinsic $v-M$ dispersion and observational uncertainties result in a scatter smaller than $20 \%$. Assuming $20 \%$ scatter is appropriate, the expected number of clusters falls within a factor of 2 of that observed for values of the bias parameter in the range $b \simeq 0.9-1.3$.

\section{b) Low-Redshift Analysis}

The same approach used above can be applied to a nearby sample. Here, we use Abell's statistical sample of 104 clusters within distance classes $D \leq 4$ and richness classes $R \geq 1$. This sample covers one-third of the sky and is complete to $z \simeq 0.1$. The heterogeneous compilation of velocity dispersions by Struble and Rood (1987) contains measurements for 62 of the 104 clusters. Of these, five are greater than $1350 \mathrm{~km} \mathrm{~s}^{-1}$ : A85 $(z=0.052, \quad v=1443), \quad \mathrm{A} 399 \quad(z=0.071, \quad v=1424), \quad \mathrm{A} 1775$ $(z=0.070, v=1539), \mathrm{A} 2029(z=0.077, v=1430)$, and A2319 $(z=0.056, v=1627)$. Abell 2319 is believed to possess significant substructure (Faber and Dressler 1977) although its X-ray temperature is among the highest known $T=12.5_{-4.0}^{+7.0} \mathrm{keV}$ (Mushotzky et al. 1978). Abell 2029 is listed by Jones and Forman (1984) as having a dispersion of only $v=1100 \mathrm{~km} \mathrm{~s}^{-1}$. The remaining three clusters are listed in Jones and Forman (1984), the data for which are unpublished.

These five clusters with $v>1350 \mathrm{~km} \mathrm{~s}^{-1}$ imply a population of $\sim 15$ such clusters covering the whole sky out to redshift $z=0.1$. Figure $3 b$ shows the predicted numbers as a function of $b$. Again, assuming $20 \%$ scatter as typical, the expected abundance falls within a factor of 2 of that observed for values of the bias parameter in the range $b \simeq 1.5-1.9$.

The abundance expected when $b=1$ is an order of magnitude greater; 50 are expected rather than five. Assuming Poisson statistics, the probability that five are observed when 50 are expected is minute, $\mathcal{O}\left(10^{-10}\right)$. Although such a dramatic statistical statement is unwarranted given the present uncertainties, it appears fair to say that the low-redshift data do not support the value of $b \simeq 1$ inferred from the high-redshift observations.

\section{DISCUSSION}

For the cold dark matter model to retain its attractive ability to reproduce observed small-scale structure, it is necessary to have a substantial level of bias $b \simeq 1.5-2.5$. Gunn and Dressler's observations of a few high-velocity dispersion systems discovered over a very small portion of the sky seem to require an unbiased $C D M$ spectrum $b \simeq 1$. However, an unbiased model is discounted by nearby cluster abundances, which instead favor moderate biasing $b \simeq 1.5-1.9$. This inconsistency prevents firm conclusions regarding the level of bias from being deduced from rich cluster observations at the present time.

There are several ways to interpret this finding. One possibility is that the high-redshift cluster dispersions are overestimated either because of contamination by projected galaxies not physically residing within the relaxed cluster volume or because the cluster as a whole has not yet relaxed and pos-

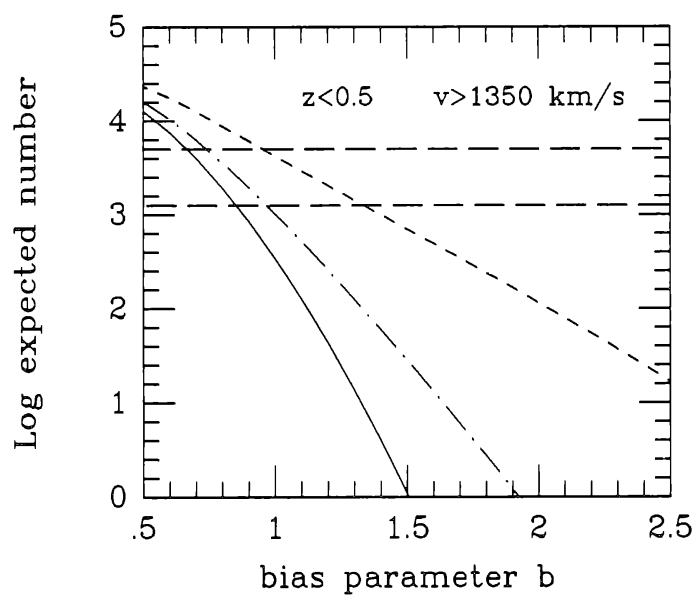

(a)

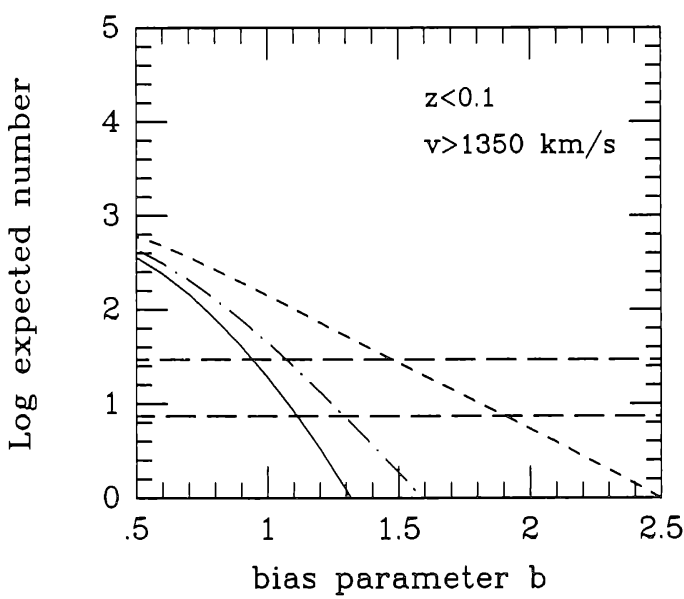

(b)

FIG. 3.-The number of clusters expected to be observed with velocity dispersion $v>1350 \mathrm{~km} \mathrm{~s}^{-1}$ is shown as a function of the bias parameter $b$ for (a)

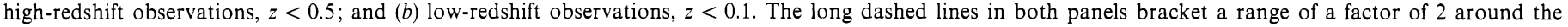
abundances inferred from observations. See the text for a discussion. 
sesses significant substructure. The observed trend in preferred $b$ values between high and low $z$ can be understood if the systematic errors associated with these effects increase with redshift. Another possible interpretation of the results is that we are seeing a fluctuation spectrum different from that of CDM, and so the theory is wrong. Without better statistics, there is simply no way to tell.

Frenk (1989) has performed a different analysis on nearby clusters. He attempts to recover the full distribution function $\mathscr{N}(v)$ over a range of $v$ from present data. This is not an easy task, as there is no statistically complete sample of cluster velocity dispersions. The shape of his derived observed velocity distribution is not reproduced by model estimates for any single value of $b$. Instead, the abundance of high-velocity clusters is matched by $b \simeq 1.6$ while data below $v=1000 \mathrm{~km} \mathrm{~s}^{-1}$ indicate $b \geq 2$. This trend probably reflects sampling incompleteness - a few rich systems will attract more attention from observers than will more sedate, but more numerous, low-dispersion clusters. For this reason, I have concentrated my analysis on the high-velocity tail of the distribution. The chosen cutoff of $1350 \mathrm{~km} \mathrm{~s}^{-1}$ is special only in that it arose naturally from a break in the sparse high-redshift data--of seven clusters listed by Gunn (1989), four were above this value. The highest dispersion of the remaining three was only $590 \mathrm{~km} \mathrm{~s}^{-1}$.
An alternative approach which would avoid many of the pitfalls associated with contamination by projected galaxies would be to use the X-ray temperature of the intracluster medium as a measure of potential well depth instead of velocity dispersion. The velocity cutoff used here corresponds to an X-ray temperature $T_{x} \simeq 9.1 \mathrm{keV}$. Unfortunately, present data on X-ray temperatures are even more incomplete than those for velocity dispersions. The upcoming all-sky survey by the ROSAT satellite will provide only an indirect measure of temperature abundances, since the satellite will carry no sensitive spectroscopic instruments. Eventually, AXAF could make follow-up observations to determine X-ray temperatures for the distant clusters expected be identified in the ROSAT survey. Unfortunately, these data are several years off, and perhaps the biased CDM theory will not survive until that time. However, the approach taken here can be applied to the general class of hierarchical clustering from Gaussian initial conditions. Constraints on the amplitude and perhaps the shape of the fluctuation spectrum could be placed with a sufficiently sensitive, complete sample of X-ray temperatures.

This work was supported by The Miller Foundation for Basic Research in Science at the University of California, Berkeley.

\section{REFERENCES}

Bardeen, J. M., Bond, J. R., Kaiser, N., and Szalay, A. S. 1986, Ap. J., 304, 15. Bertschinger, E., and Juszkiewicz, R. 1988, Ap. J. (Letters), 334, L59.

Davis, M., Efstathiou, G., Frenk, C. S., and White, S. D. M. 1985, Ap. J., 292, 371.

Davis, M., and Peebles, P. J. E. 1983, Ap. J., 267, 465.

Efstathiou, G., Frenk, C. S., White, S. D. M., and Davis, M. 1988, M.N.R.A.S., 234, 715 .

Evrard, A. E. 1989a, in Large-Scale Structure and Motions in the Universe, ed. G. Giuricin et al. (Dordrecht: Reidel), in press. . 1989b, Ap. J., 341, 26.

. 1989c, in preparation.

Faber, S., and Dressler, A. 1977, A.J., 82, 187.

Frenk, C. S. 1989, in The Epoch of Galaxy Formation, ed. R. Ellis and C. Frenk (Dordrecht: Kluwer), in press.

Frenk, C. S., White, S. D. M., Davis, M., and Efstathiou, G. 1988, Ap. J., 327, 507.
Gorski, K., Davis, M., Strauss, M. A., White, S. D. M., and Yahil, A. 1989, Ap. $J$., in press.

Gunn, J. E. 1989, in The Epoch of Galaxy Formation, ed. R. Ellis and C. Frenk (Dordrecht: Kluwer), in press.

Gunn, J. E., and Gott, J. R. 1972, Ap. J., 209, 1.

Gunn, J. E., Hoessel, J. G., and Oke, J. B. 1986, Ap. J., 306, 30.

Jones, C., and Forman, W. 1984, Ap. J., 276, 38.

Kaiser, N., and Lahav, O. 1989, M.N.R.A.S., in press

Koo, D. C., and Kron, R. G. 1987, in IAU Symposium 124, Observational Cosmolology, ed. A. Hewitt, G. Burbridge, and L. Z. Fang (Dordrecht: Reidel), p. 383.

Mushotzky, R. F., Serlemitsos, P. J., Smith, B. W., Boldt, E. A., and Holt, S. S. 1978, Ap. J., 225, 21.

Press, W. H., and Schechter, P. 1974, Ap. J., 187, 425.

Struble, M. F., and Rood, H. J. 1987, Ap. J. Suppl., 63, 543. 